\title{
Interventional Molecular Imaging
}

\author{
Stephen B. Solomon ${ }^{1}$ and Francois Cornelis ${ }^{1,2}$ \\ ${ }^{1}$ Department of Radiology, Memorial Sloan Kettering Cancer Center, New York, New York; and ${ }^{2}$ Department of Radiology, Pellegrin \\ Hospital, Bordeaux, France
}

\begin{abstract}
Although molecular imaging has had a dramatic impact on diagnostic imaging, it has only recently begun to be integrated into interventional procedures. Its significant impact is attributed to its ability to provide noninvasive, physiologic information that supplements conventional morphologic imaging. The four major interventional opportunities for molecular imaging are, first, to provide guidance to localize a target; second, to provide tissue analysis to confirm that the target has been reached; third, to provide in-room, posttherapy assessment; and fourth, to deliver targeted therapeutics. With improved understanding and application of ${ }^{18} \mathrm{~F}-\mathrm{FDG}$, as well as the addition of new molecular probes beyond ${ }^{18} \mathrm{~F}-\mathrm{FDG}$, the future holds significant promise for the expansion of molecular imaging into the realm of interventional procedures.
\end{abstract}

Key Words: molecular imaging; cancer; interventional oncology; biopsy; ablation; interventional radiology; PET/CT; fluorescence imaging

J Nucl Med 2016; 57:493-496

DOI: $10.2967 /$ jnumed.115.161190

With the advent of each new diagnostic imaging tool-from radiography to ultrasound to CT to MRI-interventionalists have been able to incorporate the added modality into their practice, leveraging its advantages and advancing the field of image-guided intervention $(1,2)$. It is therefore not surprising that molecular imaging is finding its way into the interventionalist's armamentarium.

Although molecular imaging has had a dramatic impact on diagnostic imaging (3), it has only recently begun to be integrated into interventional procedures. Its significant impact is attributed to its ability to provide noninvasive, physiologic information that supplements conventional morphologic imaging (4). With improved understanding and application of ${ }^{18} \mathrm{~F}-\mathrm{FDG}$, as well as the addition of new molecular probes beyond ${ }^{18} \mathrm{~F}-\mathrm{FDG}$, the future holds significant promise for the expansion of molecular imaging into the realm of interventional procedures (5).

Molecular imaging probes are based on radioisotopes, fluorescence, or combinations of these (4). Because most interventional radiology procedures are imaged extracorporeally and most fluorescent probes have only a limited depth of penetration (6), the field of molecular imaging for interventional radiologists currently is based mostly on molecular probes using radioisotopes, usually ${ }^{18} \mathrm{~F}$-FDG PET (7). In the future, as integration of PET scanners and optical imaging scopes takes place, use of combination molecular probes may become more common (8).

Received Sep. 24, 2015; revision accepted Feb. 9, 2016.

For correspondence or reprints contact: Stephen B. Solomon, Department of Radiology, Memorial Sloan Kettering Cancer Center, 1275 York Ave., New York, NY 10065.

E-mail: solomons@mskcc.org

Published online Feb. 11, 2016.

COPYRIGHT (C 2016 by the Society of Nuclear Medicine and Molecular Imaging, Inc.
With the advantages of having high specificity and providing physiologic information, especially when coupled with anatomic imaging such as in PET/CT, molecular imaging has become an invaluable tool for the interventionalist. The four major interventional opportunities for molecular imaging are, first, to provide guidance to localize a target; second, to provide tissue analysis to confirm that the target has been reached; third, to provide in-room, posttherapy assessment; and fourth, to deliver targeted therapeutics. This article will provide an update on the status of interventional molecular imaging.

\section{FUSION OF PREVIOUS IMAGES WITH PROCEDURAL IMAGES}

Previously acquired PET images can be fused or registered with intraprocedural CT or ultrasound images to integrate the physiologic information from ${ }^{18} \mathrm{~F}$-FDG PET with the detailed anatomic information from the cross-sectional techniques (9). The usefulness of this multimodality fusion has been demonstrated for both image-guided biopsies and tumor ablations $(10,11)$. However, this technique is limited by the need to use previously obtained PET images and the difficulty of registration (12).

Challenges to registering previously acquired images with procedural images include differences in patient positioning between the previous imaging session and the procedural imaging session (e.g., procedures may be performed with the patient prone, lateral, or oblique), differences in respiration, differences in arm positioning, and differences resulting from instruments pushing tissue during a procedure (13). These challenges have led some to perform PET-guided procedures within a PET/CT suite, where improved registration is possible $(13,14)$.

\section{DEDICATED INTERVENTIONAL PET/CT SCANNERS}

A dedicated interventional PET/CT scanner would be ideal to avoid several of the image registration issues. With such a scanner, the position of the patient during the procedural PET scan would be identical to that during the procedural CT scan. With assistance from the anesthesia team, registration issues stemming from respiratory motion may be reduced (13). Although continuous breathing during diagnostic PET acquisitions may distort lesion size or shape or create partial-volume effects, particularly along the direction of maximal respiratory motion, registration may be improved through the use of breath-holding techniques, sedation, or general anesthesia during the procedure $(15,16)$. The use of short-breath-hold PET acquisitions (e.g., 20 s) has been shown valuable (13). Also, performing a procedure within the PET scanner allows updating of the PET data at different time points in response to procedure-related changes.

\section{INTERVENTION-CUSTOMIZED ACQUISITION PROTOCOLS}

Although diagnostic imaging is focused on obtaining the highestquality images, interventional imaging is focused much more on rapidly obtaining the information needed to perform the procedure at hand (13). Because patients always have a diagnostic-quality study available for planning, the interventional imaging can be of lower quality and tailored to meet the needs of the procedure. For example, interventional 
PET/CT is usually performed on only one or two focused bed positions rather than on the whole body. Instead of using the standard 444$\mathrm{MBq}{ }^{18} \mathrm{~F}-\mathrm{FDG}$ dose that is applied for diagnostic studies, in our practice we use about $222 \mathrm{MBq}$ to guide biopsies and about $148 \mathrm{MBq}$ to guide ablations. The ${ }^{18}$ F-FDG uptake times are usually quite variable and depend on the patient preparation and positioning times. In our experience, the uptake times have ranged from 45 to $160 \mathrm{~min}$. Even with extended uptake times, imaging of sufficient quality has been achieved (17). The PET acquisition times are usually lowered to the length of a single breath hold, which may be $20 \mathrm{~s}$ (13). If general anesthesia and paralysis are used, 90-s acquisition times with respiratory suspension can be applied to increase image quality and improve registration (15).

During PET/CT-guided interventions, a PET image acquired at the start of the procedure can rapidly be fused with multiple series of CT images acquired as the needle advances toward the target. PET imaging can also be repeated to confirm that the target has been reached.

When the split-dose technique is used to assess an interventional treatment such as an ablation, an initial $148-\mathrm{MBq}{ }^{18} \mathrm{~F}-\mathrm{FDG}$ dose is used to guide the needle and a second dose of about $222 \mathrm{MBq}$ is used after ablation to assess the completeness of treatment (Fig. 1) (14). The uptake time allowed after the second dose is approximately $30 \mathrm{~min}$ rather than the $60 \mathrm{~min}$ usually allowed for diagnostic PET/CT. All these protocol modifications emphasize the differing priorities of interventional versus diagnostic imaging.

\section{THE INTERVENTIONAL PET/CT ROOM}

Performing interventions within a PET/CT scanner is both feasible and valuable (7) and can be made more practical through certain modifications (15). These include CT fluoroscopy, an in-room image display, an anesthesia boom with gases, and a system to communicate between the control room and the procedure room. A scanner with a large bore $(>70 \mathrm{~cm})$ to accommodate the patient plus a partially extending needle is critical.

\section{CLINICAL APPLICATIONS}

\section{Guidance of Biopsy}

Targeted percutaneous biopsies are commonly performed under CT and ultrasound guidance to obtain a definitive tissue diagnosis. For ${ }^{18} \mathrm{~F}-\mathrm{FDG}-$ avid lesions, PET/CT guidance has been shown to enable targeting of lesions that are not well visualized with $\mathrm{CT}$ or other imaging methods (Fig. 2) (18). A particular advantage of ${ }^{18} \mathrm{~F}-\mathrm{FDG}$ PET/CT guidance is the ability to differentiate and selectively sample the most metabolically active component of a heterogeneous lesion (19). This capability may reduce false-negative results and improve disease stratification (20). For example, it is known that hypermetabolic, ${ }^{18} \mathrm{~F}-\mathrm{FDG}$-avid regions within neurofibromas may be areas of malignant transformation (21). It is important, therefore, that these hypermetabolic areas be targeted for biopsy. Biopsy is commonly used to detect cancer recurrence after surgery, radiation therapy, or ablation. When based simply on anatomic images, such biopsies can be especially confounded by prior treatment. PET/CT can guide the biopsy to the metabolically active area in a previously treated zone (22). Another advantage of PET/CT is that it may be able to demonstrate abnormalities not visible on anatomic images. Performing these procedures in a PET/CT suite permits confirmation of reaching the target.

\section{Validation of New Molecular Tracers}

As new PET tracers beyond ${ }^{18} \mathrm{~F}-\mathrm{FDG}$ are developed to leverage increased target specificity, their imaging findings need pathologic validation by PET/CT-guided biopsy. For example, guided biopsy of PETpositive sites has been used to confirm the sensitivity and specificity of two tracers of prostate-specific membrane antigen: the monoclonal antibody tracer ${ }^{89} \mathrm{Zr}$-huJ591 (23) and the small-peptide tracer ${ }^{18} \mathrm{~F}$-DCFPyL (24). In the case of PET imaging of angiogenesis with the tracer ${ }^{18} \mathrm{~F}$-alfatide, tissue confirmation through guided biopsy demonstrated the difficulty of distinguishing inflammation from cancer (25).

\section{Confirmation of Biopsy Adequacy}

To be certain that a needle-biopsy specimen contains sufficient tissue to allow for a diagnosis, a pathologist is frequently asked to make an on-site evaluation. For ${ }^{18} \mathrm{~F}$-FDG-avid lesions, such a determination may be possible with an on-site autoradiography device (26). Fanchon et al. found that on-site autoradiographic measurement of a biopsy specimen could determine the probability that it would be positive for cancer (26). This type of system might be applicable when there is no PET scanner to guide the intervention; with injection of a known level of radioactivity, the likelihood of appropriate biopsy targeting can be determined even without a PET scanner simply by measuring for the presence of a threshold level of radioactivity. Similar systems that use fluorescent molecular probes have also been applied to analyze biopsy specimens for adequacy (6).

\section{Assessment of Treatment}

Several studies have shown the value of imaging for detecting recurrence of tumor several months after ablation (5). However, early detection of recurrence with ${ }^{18} \mathrm{~F}-\mathrm{FDG}$ PET has been confounded by ${ }^{18} \mathrm{~F}-\mathrm{FDG}$ uptake related to inflammation and posttherapy changes (27). The novel use of ${ }^{18}$ F-FDG PET immediately after an ablation, while the patient is still on the interventional table, offers an opportunity to detect viable cells before inflammation begins and to administer additional treatment before the end of the procedure.

In one approach, dubbed the split-dose technique (14), the standard 444-MBq ${ }^{18} \mathrm{~F}-\mathrm{FDG}$ dose for diagnostic PET/CT is split into two doses. The first dose, $148 \mathrm{MBq}$, is administered before the procedure to guide placement of the ablation probes. The second dose, $296 \mathrm{MBq}$, is given after the ablation to assess for viability. Although the first dose remains trapped in the ablated cells (28), it has decayed significantly by the time the second dose is given. The ratio of the initial ${ }^{18} \mathrm{~F}-\mathrm{FDG}$ in the patient (i.e., the background radioactivity) to the new ${ }^{18} \mathrm{~F}$-FDG is approximately 1:7. We have used this ratio to assess for areas of viable cancer after ablation of liver tumors. To normalize ablation-zone uptake for background uptake, we calculate the tissue radioactivity concentration (TRC) ratio as follows: $\mathrm{TRC}$ ratio $=\left[\right.$ (lesion $\mathrm{TRC}_{\max }-$ liver $\left.\mathrm{TRC}_{\text {mean }}\right) /$ lesion $\left.\mathrm{TRC}_{\mathrm{max}}\right] \times 100$. In one series of patients from our institution, a positive predictive value of $100 \%$ was found by calculating the TRC in the treatment zone (TRC ratio $>28.3$ ) (Fig. 3). The sensitivity of this method of detecting residual disease immediately after ablation is better than that of contrast-enhanced CT. It is hoped that this use of molecular imaging to assess and improve an interventional treatment before the end of the procedure will lead to improved outcomes.

\section{RADIOTRACER CHALLENGES}

Although the use of ${ }^{18} \mathrm{~F}-\mathrm{FDG}$ has enabled exploration of interventional applications of molecular imaging, ${ }^{18} \mathrm{~F}-\mathrm{FDG}$ has limitations due to its biodistribution and half-life (15). The 110 -min half-life of ${ }^{18} \mathrm{~F}$ means that treatment can be effectively assessed only once during a procedure. The split-dose approach with a long procedure time allows for a potential second dose. However, a tracer with a shorter half-life (such as ${ }^{11} \mathrm{C}$ ) would be more ideal, as it would allow for multiple doses and assessments throughout the treatment procedure without the confounding effect of remaining activity from earlier doses (29).

\section{RADIATION EXPOSURE}

Interventionalists must be aware that the factors governing radiation exposure in PET/CT are different from those most familiar 


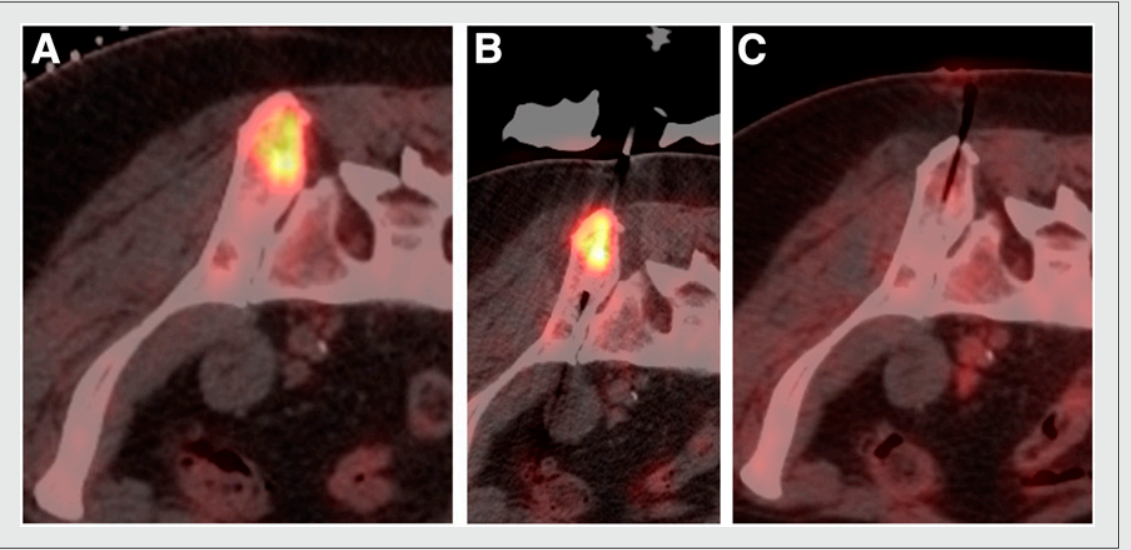

FIGURE 1. Split-dose technique for tumor ablation in 63-y-old man with solitary esophageal cancer metastasis to left posterior iliac bone that was visible on ${ }^{18} \mathrm{~F}-\mathrm{FDG}$ PET/CT but not on CT alone. (A) Initial 148-MBq ${ }^{18} \mathrm{~F}-\mathrm{FDG}$ PET/CT scan demonstrated the lesion. (B) PET/CT guided placement of cryoablation needle. (C) After ablation and with patient still on interventional table, an additional $296 \mathrm{MBq}$ of ${ }^{18} \mathrm{~F}-\mathrm{FDG}$ were injected and showed no residual ${ }^{18} \mathrm{~F}-\mathrm{FDG}$ activity. Track in bone depicts prior location of needle.

to interventional radiologists (15). In fluoroscopy- or CT-guided procedures, the operator controls the radiation dose directly by controlling the $\mathrm{x}$-ray source and can limit the exposure time; increase the distance from the patient, when feasible; use low-dose, collimation, and antiscatter techniques; and wear protective devices such as shields. Because the source of the radiation is the patient, and because the photon energy of PET is $511 \mathrm{keV}$ higher than the typical x-ray photon energy of fluoroscopy (70-120 keV), personal protective devices are relatively ineffective against PET radiation.

However, the dose to the operator from PET/CT-guided procedures is not significantly different from typical doses from fluoroscopically guided procedures (30). Ryan et al. (30) showed that the median

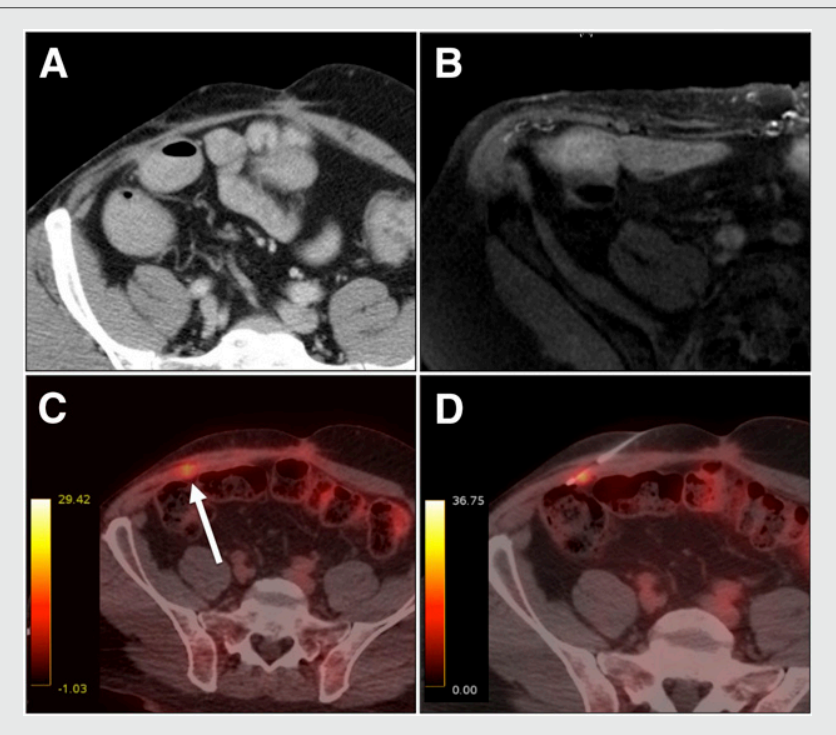

FIGURE 2. PET-guided percutaneous biopsy in 50-y-old man with metastatic colorectal cancer and rising level of carcinoembryonic antigen. (A-C) Although no disease was seen on contrast-enhanced CT (A) or MR (B), one site of disease was visible on ${ }^{18} \mathrm{~F}$-FDG PET/CT (arrow, C). (D) Percutaneous biopsy was then performed under PET guidance (148 MBq of ${ }^{18} \mathrm{~F}-\mathrm{FDG}$; injection-to-scan time, $\left.47 \mathrm{~min}\right)$. Pathologic evaluation of biopsy sample found metastasis of colorectal carcinoma. effective dose during an ablation in which $444 \mathrm{MBq}$ of ${ }^{18} \mathrm{~F}-\mathrm{FDG}$ were injected was $0.02 \mathrm{mSv}$ (range, $0-0.13 \mathrm{mSv}$ ) for the primary operator, $0.01 \mathrm{mSv}$ (range, $0-0.05 \mathrm{mSv}$ ) for the nurse anesthetist, and $0.02 \mathrm{mSv}$ (range, 0 $0.05 \mathrm{mSv}$ ) for the radiology technologist. The median extremity-dose equivalent for the operator was $0.05 \mathrm{mSv}$ (range, $0-0.62 \mathrm{mSv}$ ). Although the median dose from PET/CTguided procedures remained within an acceptable range for an interventional radiologist, the range of doses in this study was varied and had a maximum of $0.13 \mathrm{mSv}$, which is higher than the median dose by a factor of 6.5 . In the study of Ryan et al., the exposure from 444-MBq dosing correlated with procedure duration and with type of imaging guidance. The median effective dose to the operator from the procedure was $0.015 \mathrm{mSv}$ with conventional biopsy-mode CT imaging guidance, compared with $0.06 \mathrm{mSv}$ with in-room imaging guidance, although the small sample size prevented this difference from achieving statistical significance $(P=0.06)$. Therefore, the major determinant of radiation exposure to the operator from PET/CT-guided interventional procedures is time spent near the patient. Given the reasonable range of exposures seen, the perception of increased radiation dose to operator and staff should not limit the expansion of PET/CT as a tool to guide intervention. Rather, different methods of minimizing radiation exposure should be emphasized during PET-guided intervention. In our practice, we have lowered the dose to $222 \mathrm{MBq}$ for biopsy and $148 \mathrm{MBq}$ for needle placement during ablation-reductions that should markedly decrease the radiation exposure of staff. In the splitdose technique, the operator is generally not closely exposed to radiation during injection of the second dose or during its uptake time.

\section{FLUORESCENT IMAGING}

Fluorescent probes have the benefit of not using ionizing radiation, but they have a limited depth of penetration (31). The application of fluorescent probes has thus been confined to optical imaging scopes that can directly visualize tissue. In one such application, the scope is passed through an introducer cannula during a biopsy, with the appearance of indocyanine green fluorescence confirming that the target has been reached. The importance of this type of molecular imaging will increase as it begins to play a larger role in interventional radiology (6).

\section{DELIVERY OF MOLECULAR THERAPEUTICS}

Interventionalists perform endovascular oncologic procedures to deliver lethal payloads directly to the vascular supply feeding cancers, thereby minimizing systemic exposure to these toxic therapeutics. Applying similar principles, Kratochwil et al. have been delivering molecularly targeted therapeutic agents to cancers (32). Relying on the increased vascularity and increased somatostatin receptor expression of neuroendocrine tumors, this group has been delivering $\alpha$-radionuclides ( ${ }^{213} \mathrm{Bi}$-DOTATOC) and $\beta$-radionuclides $\left({ }^{90} \mathrm{Y}\right.$ - or ${ }^{177} \mathrm{Lu}$-DOTATOC) with good clinical effect and limited toxicity (33).

\section{COST-EFFECTIVENESS}

Just as with every new technology, be it diagnostic PET/MR, optical cameras, or interventional PET/CT, cost and value need to be addressed. Dedicating a PET/CT scanner to interventional applications has been shown to have the value of allowing biopsies of targets not visualized on 


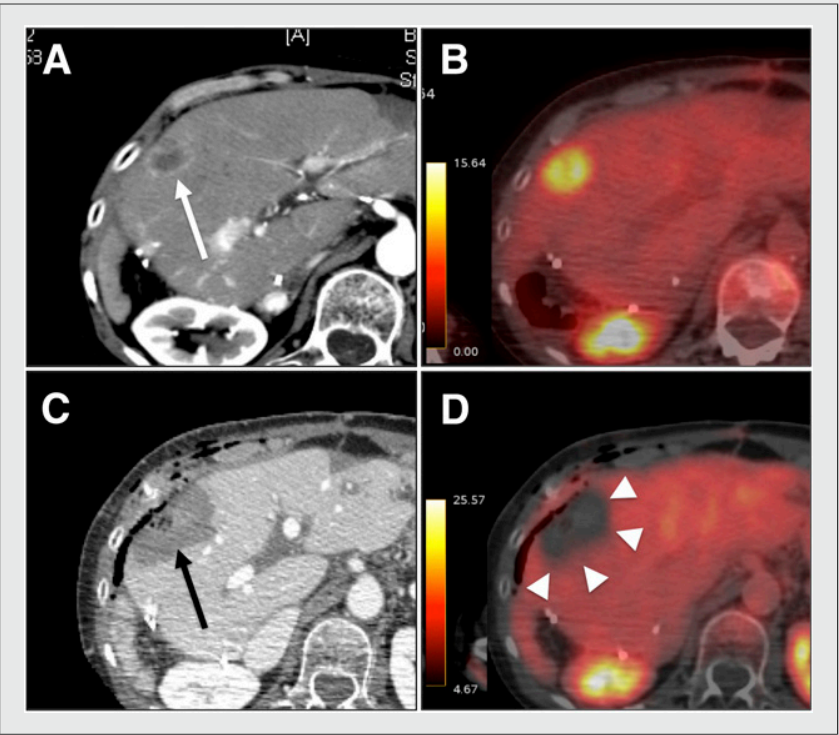

FIGURE 3. Immediate postablation treatment assessment with intraprocedural split-dose PET/CT in 65-y-old woman with metastatic colorectal cancer and rising level of carcinoembryonic antigen after partial liver resection. ( $A$ and B) Liver metastasis was seen on contrast-enhanced CT (white arrow, A) and ${ }^{18} \mathrm{~F}-\mathrm{FDG} \mathrm{PET}(\mathrm{B})$ before ablation (148 MBq of ${ }^{18} \mathrm{~F}-\mathrm{FDG}$; injection-to-scan time, $52 \mathrm{~min}$ ). (C) After ablation, ablation zone was seen as defect on contrastenhanced CT (black arrow). (D) After reinjection of ${ }^{18} \mathrm{~F}-\mathrm{FDG}$ (296 MBq; injectionto-scan time, $37 \mathrm{~min}$ ), no activity remained within ablation zone or margins on PET/CT (arrowheads), confirming immediate efficacy of radiofrequency ablation. No in situ recurrence was observed during 12 mo of follow-up.

conventional imaging. Similarly, the possible cost value of interventional PET/CT, such as through an improvement in ablation outcomes or a reduction in the number of ablations that need to be repeated because of incomplete treatment, needs to be more formally studied.

\section{CONCLUSION}

As the role of molecular imaging in cancer diagnosis increases, the role in interventional oncology will expand as well. The physiologic information afforded by molecular imaging adds value to and complements the information provided by anatomic imaging. The ability to distinguish viable from nonviable tumors and to identify particular foci of cancer amid tumor heterogeneity are critical benefits of molecular tracers. Of the four major interventional opportunities for molecular imaging - to guide toward a target, confirm that it has been reached, assess treatment, and deliver targeted therapeutics-value for target localization and treatment assessment has already been demonstrated. Procedure protocols that leverage prior diagnostic studies permit modified, interventionally favorable techniques. However, additional work to improve hardware, tracers, and logistics is still needed. Like other imaging tools that were initially thought valuable for diagnosis and later became so for interventional procedures as well, molecular imaging clearly will become a critical adjunct in interventions.

\section{DISCLOSURE}

No potential conflict of interest relevant to this article was reported.

\section{REFERENCES}

1. Smith KA, Kim HS. Interventional radiology and image-guided medicine: interventional oncology. Semin Oncol. 2011;38:151-162.
2. Solomon SB, Silverman SG. Imaging in interventional oncology. Radiology. 2010;257:624-640.

3. McDermott S, Kilcoyne A. Molecular imaging: its current role in cancer. QJM. August 7, 2015 [Epub ahead of print].

4. Mahajan A, Goh V, Basu S, et al. Bench to bedside molecular functional imaging in translational cancer medicine: to image or to imagine? Clin Radiol. 2015;70:1060-1082.

5. McLoney ED, Isaacson AJ, Keating P. The role of PET imaging before, during, and after percutaneous hepatic and pulmonary tumor ablation. Semin Intervent Radiol. 2014;31:187-192.

6. Sheth RA, Arellano RS, Uppot RN, et al. Prospective trial with optical molecular imaging for percutaneous interventions in focal hepatic lesions. Radiology. 2015;274:917-926.

7. Samim M, El-Haddad GE, Molenaar IQ, et al. $\left[{ }^{18} \mathrm{~F}\right]$ fluorodeoxyglucose PET for interventional oncology in liver malignancy. PET Clin. 2014;9:469-495.

8. Xi L, Jiang H. Image-guided surgery using multimodality strategy and molecular probes. Wiley Interdiscip Rev Nanomed Nanobiotechnol. 2016;8:46-60.

9. Yokoyama K, Ikeda O, Kawanaka K, et al. Comparison of CT-guided percutaneous biopsy with and without registration of prior PET/CT images to diagnose mediastinal tumors. Cardiovasc Intervent Radiol. 2014;37:1306-1311.

10. Venkatesan AM, Kadoury S, Abi-Jaoudeh N, et al. Real-time FDG PET guidance during biopsies and radiofrequency ablation using multimodality fusion with electromagnetic navigation. Radiology. 2011;260:848-856.

11. Bajpai S, Kambadakone A, Guimaraes AR, Arellano RS, Gervais DA, Sahani D. Image-guided treatment in the hepatobiliary system: role of imaging in treatment planning and posttreatment evaluation. Radiographics. 2015;35:1393-1418.

12. Tatli S, Gerbaudo VH, Mamede M, Tuncali K, Shyn PB, Silverman SG. Abdominal masses sampled at PET/CT-guided percutaneous biopsy: initial experience with registration of prior PET/CT images. Radiology. 2010;256:305-311.

13. Shyn PB, Tatli S, Sahni VA, et al. PET/CT-guided percutaneous liver mass biopsies and ablations: targeting accuracy of a single $20 \mathrm{~s}$ breath-hold PET acquisition. Clin Radiol. 2014;69:410-415.

14. Ryan ER, Sofocleous CT, Schoder H, et al. Split-dose technique for FDG PET/CT-guided percutaneous ablation: a method to facilitate lesion targeting and to provide immediate assessment of treatment effectiveness. Radiology. 2013;268:288-295.

15. Shyn PB. Interventional positron emission tomography/computed tomography: state-of-the-art. Tech Vasc Interv Radiol. 2013;16:182-190.

16. Li G, Schmidtlein CR, Burger IA, Ridge CA, Solomon SB, Humm JL. Assessing and accounting for the impact of respiratory motion on FDG uptake and viable volume for liver lesions in freebreathing PET using respiration-suspended PET images as reference. Med Phys. 2014:41:091905.

17. Povoski SP, Murrey DA Jr, Smith SM, Martin EW Jr, Hall NC. ${ }^{18}$ F-FDG PET/CT oncologic imaging at extended injection-to-scan acquisition time intervals derived from a single-institution ${ }^{18} \mathrm{~F}$-FDG-directed surgery experience: feasibility and quantification of ${ }^{18} \mathrm{~F}-\mathrm{FDG}$ accumulation within ${ }^{18} \mathrm{~F}$-FDG-avid lesions and background tissues. BMC Cancer. 2014;14:453.

18. Cornelis F, Silk M, Schoder H, et al. Performance of intra-procedural 18-fluorodeoxyglucose PET/CT-guided biopsies for lesions suspected of malignancy but poorly visualized with other modalities. Eur J Nucl Med Mol Imaging. 2014;41:2265-2272.

19. Klaeser B, Wiskirchen J, Wartenberg J, et al. PET/CT-guided biopsies of metabolically active bone lesions: applications and clinical impact. Eur J Nucl Med Mol Imaging. 2010;37:2027-2036.

20. Guralnik L, Rozenberg R, Frenkel A, Israel O, Keidar Z. Metabolic PET/CT-guided lung lesion biopsies: impact on diagnostic accuracy and rate of sampling error. J Nucl Med. 2015;56:518-522.

21. Chirindel A, Chaudhry M, Blakeley JO, Wahl R. ${ }^{18} \mathrm{~F}$-FDG PET/CT qualitative and quantitative evaluation in neurofibromatosis type 1 patients for detection of malignant transformation: comparison of early to delayed imaging with and without liver activity normalization. $J \mathrm{Nucl}$ Med. 2015;56:379-385.

22. Goense L, van Rossum PS, Reitsma JB, et al. Diagnostic performance of ${ }^{18} \mathrm{~F}$-FDG PET and $\mathrm{PET} / \mathrm{CT}$ for the detection of recurrent esophageal cancer after treatment with curative intent: a systematic review and meta-analysis. J Nucl Med. 2015;56:995-1002.

23. Pandit-Taskar N, O'Donoghue JA, Durack JC, et al. A phase I/II study for analytic validation of ${ }^{89} \mathrm{Zr}$-J591 immunoPET as a molecular imaging agent for metastatic prostate cancer. Clin Cancer Res. 2015;21:5277-5285.

24. Rowe SP, Gorin MA, Hammers HJ, et al. Imaging of metastatic clear cell renal cell carcinoma with PSMA-targeted ${ }^{18} \mathrm{~F}$-DCFPyL PET/CT. Ann Nucl Med. 2015;29:877-882.

25. Gao S, Wu H, Li W, et al. A pilot study imaging integrin $\alpha v \beta 3$ with RGD PET/CT in suspected lung cancer patients. Eur J Nucl Med Mol Imaging. 2015;42:2029-2037.

26. Fanchon LM, Dogan S, Moreira AL, et al. Feasibility of in situ, high-resolution correlation of tracer uptake with histopathology by quantitative autoradiography of biopsy specimens obtained under ${ }^{18} \mathrm{~F}$-FDG PET/CT guidance. J Nucl Med. 2015;56:538-544.

27. Vandenbroucke F, Vandemeulebroucke J, Ilsen B, et al. Predictive value of pattern classification 24 hours after radiofrequency ablation of liver metastases on CT and positron emission tomography/CT. J Vasc Interv Radiol. 2014;25:1240-1249.

28. Schoellnast H, Larson SM, Nehmeh SA, Carrasquillo JA, Thornton RH, Solomon SB. Radiofrequency ablation of non-small-cell carcinoma of the lung under real-time FDG PET CT guidance. Cardiovasc Intervent Radiol. 2011;34(suppl 2):S182-S185.

29. Bao A, Goins B, Dodd GD III, et al. Real-time iterative monitoring of radiofrequency ablation tumor therapy with ${ }^{15} \mathrm{O}$-water PET imaging. J Nucl Med. 2008;49:1723-1729.

30. Ryan ER, Thornton R, Sofocleous CT, et al. PET/CT-guided interventions: personnel radiation dose. Cardiovasc Intervent Radiol. 2013;36:1063-1067.

31. Chin PT, Welling MM, Meskers SC, Valdes Olmos RA, Tanke H, van Leeuwen FW. Optical imaging as an expansion of nuclear medicine: Cerenkov-based luminescence vs fluorescencebased luminescence. Eur J Nucl Med Mol Imaging. 2013;40:1283-1291.

32. Kratochwil C, Lopez-Benitez R, Mier W, et al. Hepatic arterial infusion enhances DOTATOC radiopeptide therapy in patients with neuroendocrine liver metastases. Endocr Relat Cancer. 2011;18:595-602.

33. Kratochwil C, Giesel FL, Bruchertseifer F, et al. ${ }^{213}$ Bi-DOTATOC receptor-targeted alpharadionuclide therapy induces remission in neuroendocrine tumours refractory to beta radiation: a first-in-human experience. Eur J Nucl Med Mol Imaging. 2014;41:2106-2119. 